\title{
A near-infrared tip-tilt sensor for the Keck I laser guide star adaptive optics system
}

\author{
Peter Wizinowich ${ }^{1, *}$, Roger Smith ${ }^{2}$, Roberto Biasi ${ }^{3}$, Sylvain Cetre ${ }^{1}$, Richard Dekany ${ }^{2}$, Bruno \\ Femenia-Castella ${ }^{1}$, Jason Fucik ${ }^{2}$, David Hale ${ }^{2}$, Chris Neyman ${ }^{1}$, Dietrich Pescoller ${ }^{3}$, Sam Ragland ${ }^{1}$, \\ Paul Stomski ${ }^{1}$, Mario Andrighettoni ${ }^{3}$, Randall Bartos ${ }^{2}$, Khanh Bui ${ }^{2}$, Andrew Cooper ${ }^{1}$, John Cromer $^{2}$, \\ Marcos van Dam ${ }^{4}$, Michael Hess ${ }^{1}$, Ean James ${ }^{1}$, Jim Lyke ${ }^{1}$, Hector Rodriguez ${ }^{2}$, Thomas Stalcup ${ }^{1}$ \\ ${ }^{1}$ W. M. Keck Observatory, 65-1120 Mamalahoa Hwy., Kamuela, HI 96743, USA; \\ ${ }^{2}$ California Institute of Technology, 1200 East California Blvd., Pasadena, CA 91125, USA; \\ ${ }^{3}$ Microgate, Via Stradivari 4, I-39100, Bolzano-Bozen, Italy; \\ ${ }^{4}$ Flat Wavefronts, P.O. Box 1060, Christchurch 8140, New Zealand. \\ *peterw@keck.hawaii.edu; phone 1808 881-3512; fax 1808 885-4464; www.keckobservatory.org
}

\begin{abstract}
The sky coverage and performance of laser guide star (LGS) adaptive optics (AO) systems is limited by the natural guide star (NGS) used for low order correction. This limitation can be dramatically reduced by measuring the tip and tilt of the NGS in the near-infrared where the NGS is partially corrected by the LGS AO system and where stars are generally several magnitudes brighter than at visible wavelengths. We present the design of a near-infrared tip-tilt sensor that has recently been integrated with the Keck I telescope's LGS AO system along with some initial on-sky results. The implementation involved modifications to the AO bench, real-time control system, and higher level controls and operations software that will also be discussed. The tip-tilt sensor is a H2RG-based near-infrared camera with 0.05 arc second pixels. Low noise at high sample rates is achieved by only reading a small region of interest, from 2x2 to 16x16 pixels, centered on an NGS anywhere in the 100 arc second diameter field. The sensor operates at either Ks or H-band using light reflected by a choice of dichroic beamsplitters located in front of the OSIRIS integral field spectrograph.
\end{abstract}

Keywords: adaptive optics, laser guide star, tip-tilt sensing, Keck Observatory

\section{INTRODUCTION}

The current Keck II [1] and Keck I NGS AO systems were commissioned in 1999 and 2001, respectively. The Keck II [2][3] and Keck I [4] LGS AO system, upgrades to the existing NGS AO systems, began science operations in 2004 and 2012, respectively. Through May 2014 a total of 521 refereed science papers were published based on data from the Keck AO systems, including 197 based on LGS AO data [5].

Each Keck LGS AO system includes a visible, quad cell avalanche photodiode based, tip-tilt sensor (known as STRAP) developed by Microgate and ESO. On the Keck systems tip-tilt stars as faint as R=19 can be used up to 60" off-axis. The impact of using fainter and further off-axis tip-tilt stars is to broaden the diffraction-limited core of the AO-corrected point spread function. The near-infrared (NIR) tip-tilt sensor project was intended to reduce this tip-tilt broadening and to dramatically increase the sky coverage of the Keck I LGS AO system.

\section{SYSTEM OVERVIEW}

The NIR tip-tilt system consists of the five subsystems shown in Figure 1. Four of the subsystems represent modifications to the existing Keck I AO system while the camera is a new system. The optical pickoff that sends light to the NIR camera and the camera itself are mounted on the AO bench just before OSIRIS [6] as shown in Figure 2.

OSIRIS is an integral field spectrograph (IFS) and imager. The IFS has a choice of 20, 35, 50 or 100 milli-arcsecond (mas) spatial scales with a field of view up to 4.8"x6.4", and a spectral resolution of 3800 using a Hawaii-2 detector. The imager has 20 mas pixels and a field of view of 20.4" using a Hawaii-1 detector. The IFS and imager fields are separated by $19.4 "$ center-to-center. 


\section{Operations Software System \\ - Pre-Observing Tools \\ - Observation Setup \\ - Calibration \\ - User Interface \\ - Observing Tools}

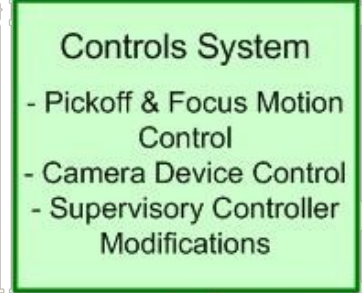

Camera System

- NIR Camera

- Camera Optics

- Camera Controller

- Filter Changer

- Cryo-cooler

- Host Computer
Opto-Mechanical System

- Pickoff Exchange Mechanism

- Focus Mechanism

- Mods to AO bench

Figure 1: Major subsystems comprising the near-infrared tip-tilt system.
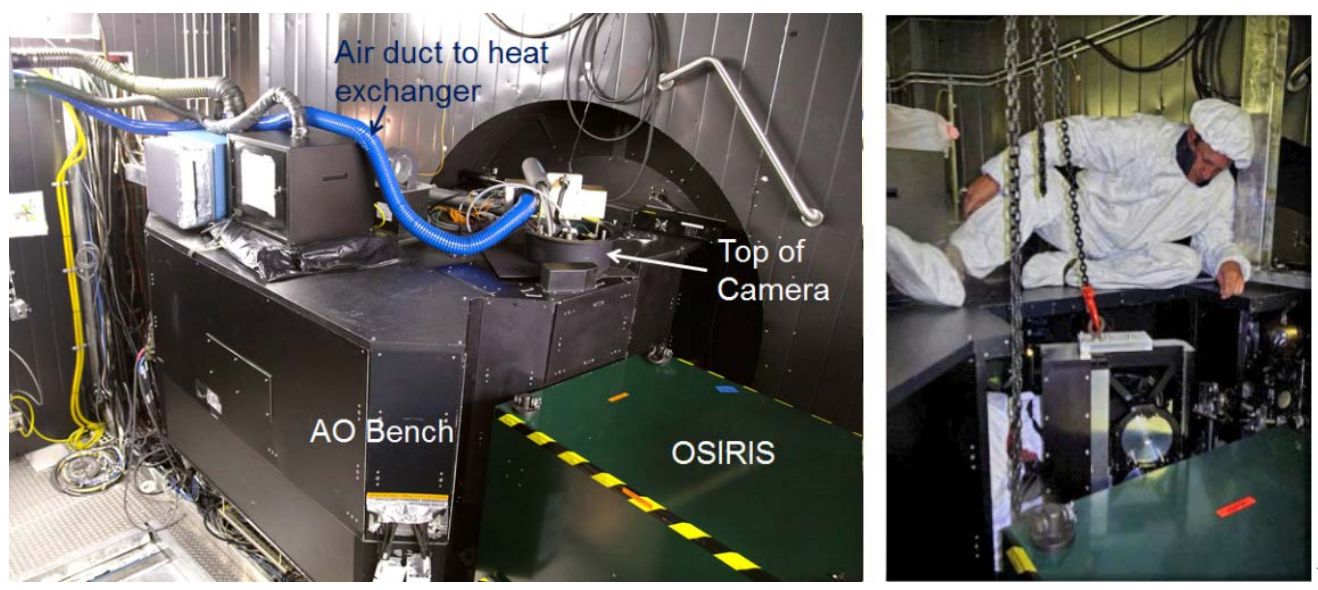

Figure 2: The near-IR tip-tilt sensor is located on the edge of the AO bench just in front of OSIRIS (left). The right image shows the optical pickoff stage, which reflects light to the near-IR tip-tilt sensor, being installed on the AO bench.

\subsection{Camera System}

The optical design of the camera is shown in Figure 3. A dichroic pickoff (part of the opto-mechanical system) is inserted in the science path $300 \mathrm{~mm}$ before the AO system's f/15 focal plane. The tilted dichroic in a converging beam introduces astigmatism that is removed by the AO system's deformable mirror. The reflected light passes through a pair of field lenses and then is reflected up by a fold mirror into the camera dewar. The dewar entrance window is tilted to match the astigmatism introduced in the science path by the dichroic pickoff. The camera 1 lens collimates the light and produces an image of the telescope pupil. The filter wheel contains two filters, each with a circular pupil stop to reduce emissivity. Camera lenses 2, 3 and 4 reimage the $\mathrm{AO}$ output focal plane onto the detector with a 50 mas/pixel plate scale. Two glasses were used for the lenses and window, $\mathrm{BaF}_{2}$ and infrasil. The camera is shown in Figure 4.

The unvignetted field of regard of the detector is shown in Figure 5 along with an image of the Galactic Center taken after the camera was integrated on the AO bench (note that NIR tip-tilt sensing is expected to provide significant benefits for LGS AO observations of the Galactic Center since a $H=9, K=7$ NGS, 6" off-axis can be used instead of a $\mathrm{R}=14$ NGS, 19" off-axis). The square edge of the field is the edge of the detector and the circular edge is due to the camera optics. The Teledyne H2RG detector in this camera is engineering grade with some anomalies. The horizontal black stripe is due to some detector rows which do not read out properly, and the bad pixels at the bottom of Figure 5 are due to some warping of the detector. The detector has also been characterized to produce bad pixel maps as a function of effective frame rate [7]. These additional bad pixels are due to pixels which are hot, low quantum efficiency, non-linear or high noise. Altogether $6.8 \%$ of the pixels are outside the camera's field of view and $10 \%$ are unusable due to detector issues. 
Multiple regions of interest (ROIs) can be read out anywhere on the detector. The camera can be read fast by only reading out small ROIs, from 2x2 to $16 x 16$ pixels per ROI. The readout time, without any requested delay, is $44 \mu$ s for $2 \times 2$ pixels and $123 \mu$ s for $4 \times 4$ pixels. Low read noise can therefore be achieved by non-destructively reading the same ROI multiple times before coadding the result as shown in Figure 6. Increasing the number of coadds reduces the read noise at the expense of reducing the tip-tilt correction bandwidth. The mean noise per pixel is $\leq 3 \mathrm{e}$ - for effective frame rates of $30 \mathrm{~Hz}$ to $200 \mathrm{~Hz}$ and rises to $\sim 4$ e- at $600 \mathrm{~Hz}$ and $\sim 5 \mathrm{e}$ - at $1 \mathrm{kHz}$ for a $4 \mathrm{x} 4$ pixel ROI at the detector operating temperature of $105 \mathrm{~K}$ [8]. Periodically the pixels are reset to avoid saturation. The camera pixel data is sent to the realtime control system over the fiber interface shown in Figure 7.

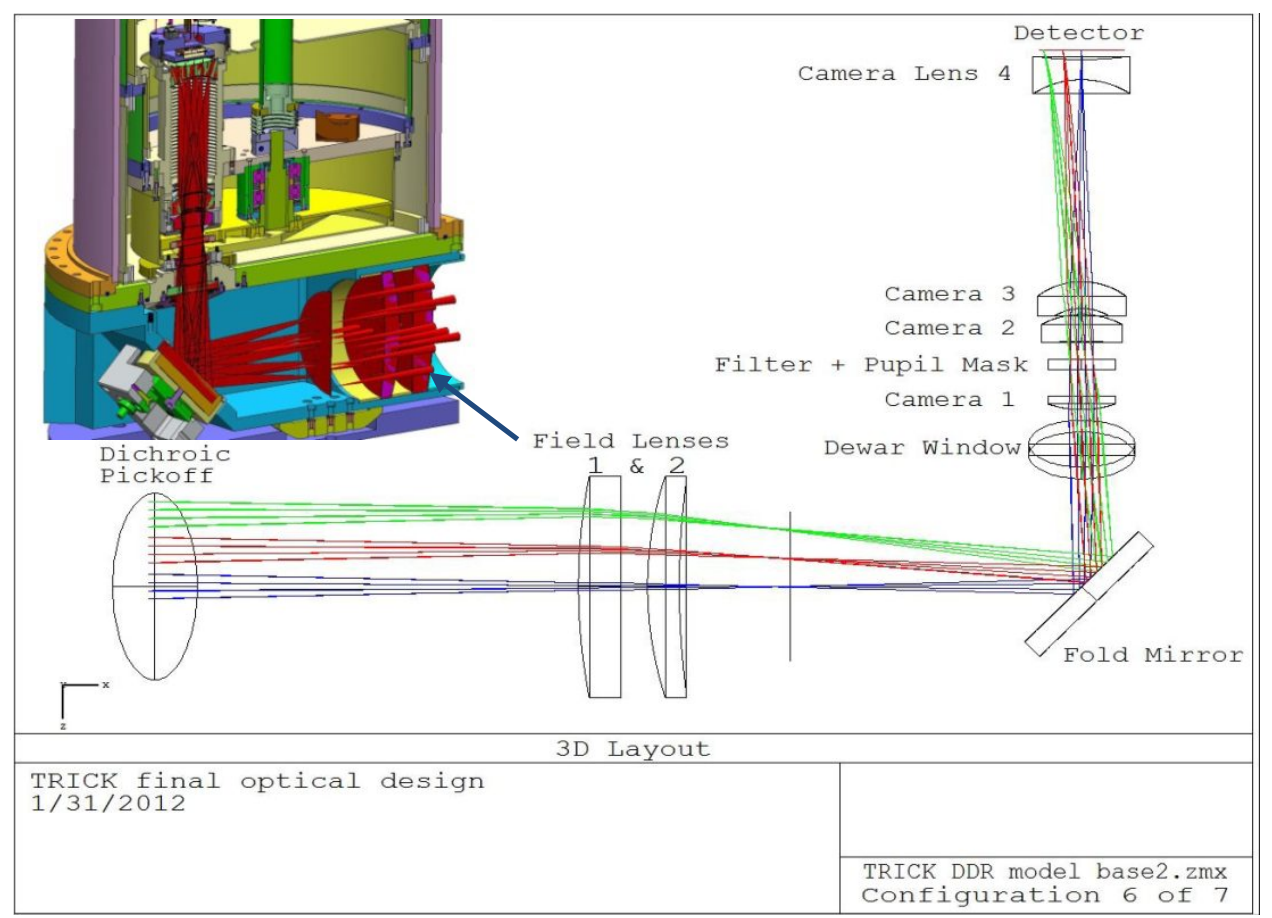

Figure 3: Optical design for the camera. The inset shows a cross-sectional view of the optics within the camera. The distance from the dichroic pickoff to the line after field lens 2 is $300 \mathrm{~mm}$.

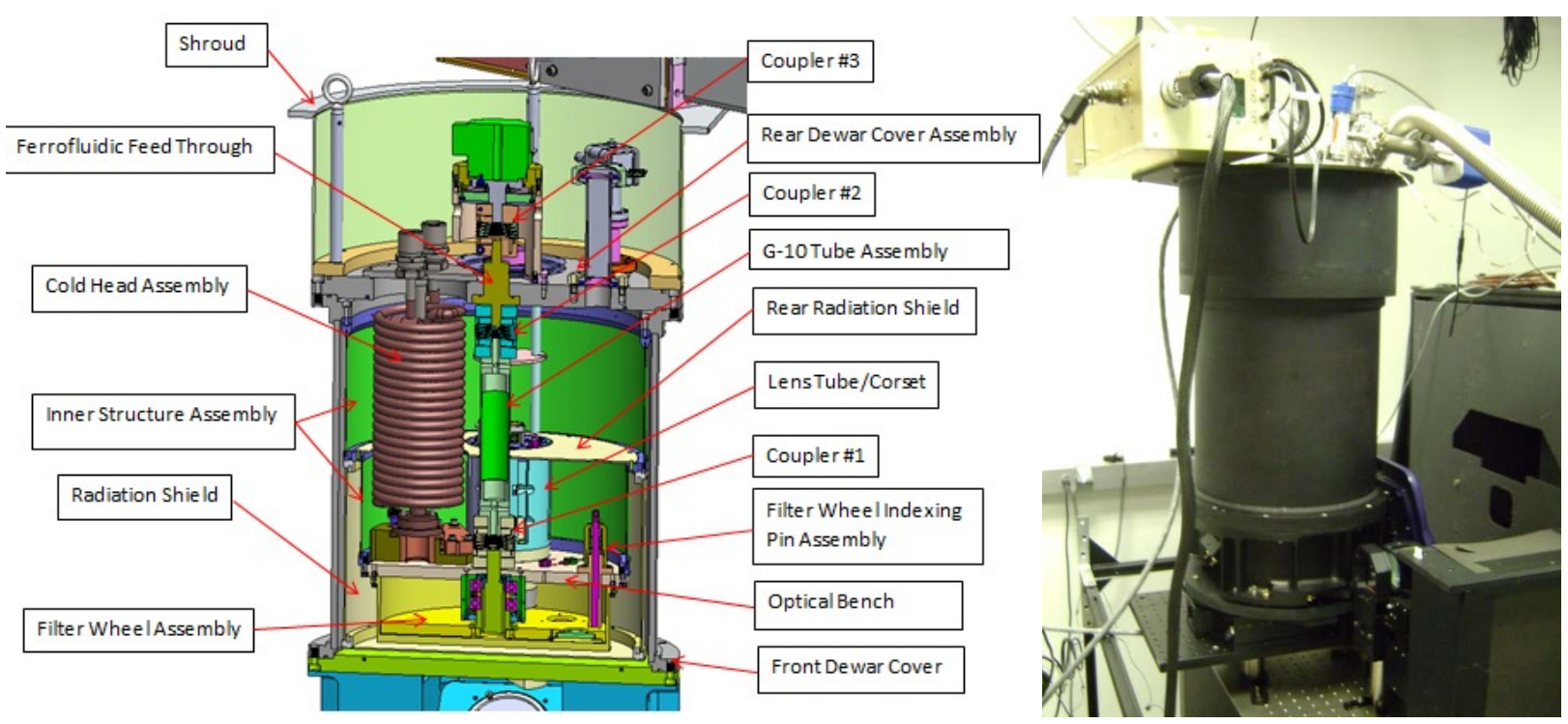

Figure 4: Cross-sectional model (of the camera (left) and a photograph of the camera (right). 


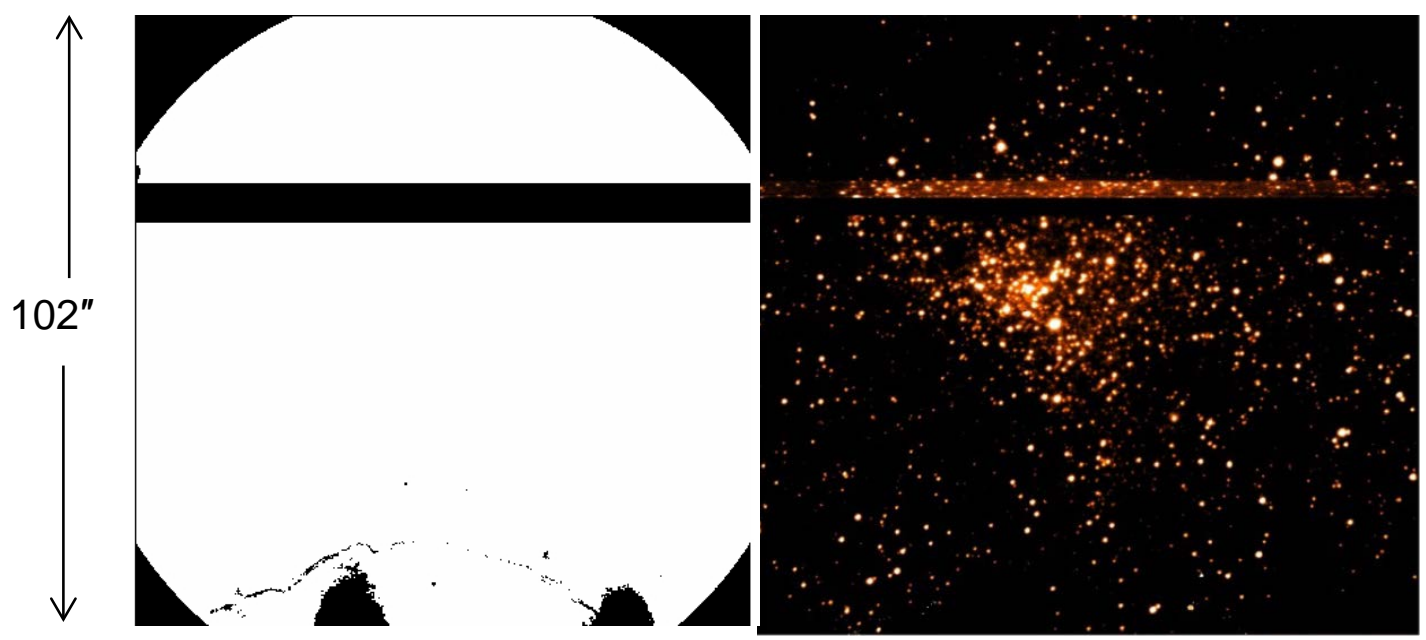

Figure 5: (Left) Camera field of regard with unavailable pixels shown in black. (Right) An H-band image of the Galactic Center taken with the camera while the tip-tilt loop was closed on STRAP.

The circular edge is due to the vignetting by the camera optics. Bad pixels include a 130 row dead band due to a multiplexer defect and some clustered cosmetic defects near the bottom.

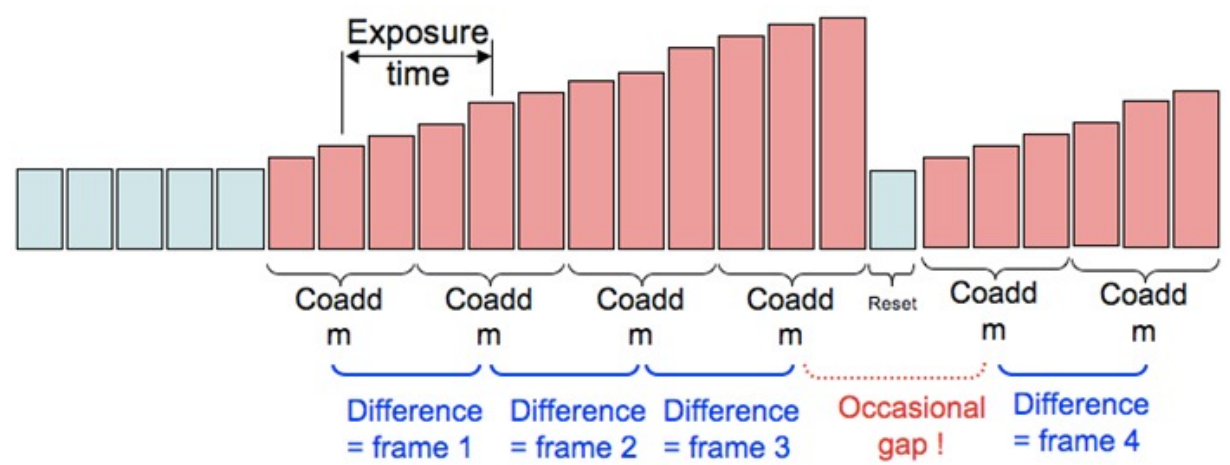

Figure 6: Camera readout approach.

Each pink bar represents a non-destructive read. In the illustrated case three reads are coadded by the real-time control system. The camera must be reset periodically to ensure that the detector full well capacity is not exceeded.

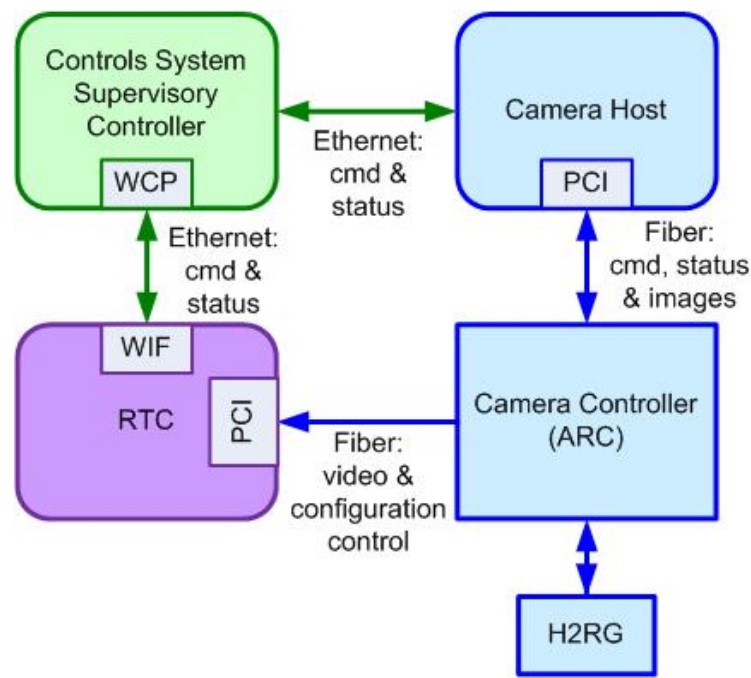

Figure 7: Communication interfaces for the camera. 


\subsection{Real-time Control System}

The existing Microgate real-time control (RTC) system hardware was modified by Microgate to support the fiber interface shown in Figure 7. The software was modified to support the real-time processing steps shown in Figure 8 . The operations software tells the camera which ROIs to read out and how many camera readout cycles to perform before resetting the camera. The processing steps can be performed for multiple ROIs. The first step (step 1 in Figure 8) is to select whether to use STRAP or the NIR tip-tilt sensor (TTS), or a combination of the two. Step 2 is to coadd the selected number of reads (" $m$ " in Figure 6) and to subtract the previous packet of coadds. The next steps (3 to 5) are to subtract the background, multiply by a flat field that is also used to remove bad pixels by setting them to zero, and to apply minimum and maximum thresholds. The centroid can be calculated using a centroid or correlation algorithm (step 6) using a reference image (step 7) in the correlation case. A centroid offset is then subtracted. The centroid offset (step 9) can be used to position the science object to a fraction of a pixel on the science camera and to maintain that position as the differential atmospheric refraction changes between the science and tip-tilt sensor wavelengths. As differential atmospheric refraction grows the ROI location may need to change (step 8) and the centroid offset should change with the location change. The interaction matrix is used for coordinate conversion (step 10) and the weighting matrix (step 11) is used to weight the relative contribution of multiple ROIs and/or tip-tilt sensors, before sending the resultant tip-tilt (TT) error to the TT controller. An algorithm can be used to calculate focus (step 12) from an ROI if there is some diagonal astigmatism in the image to the ROI. Although the camera and RTC have been implemented to fully support Figure 8, only single ROI tip-tilt sensing operation has been demonstrated on-sky prior to submitting this paper.
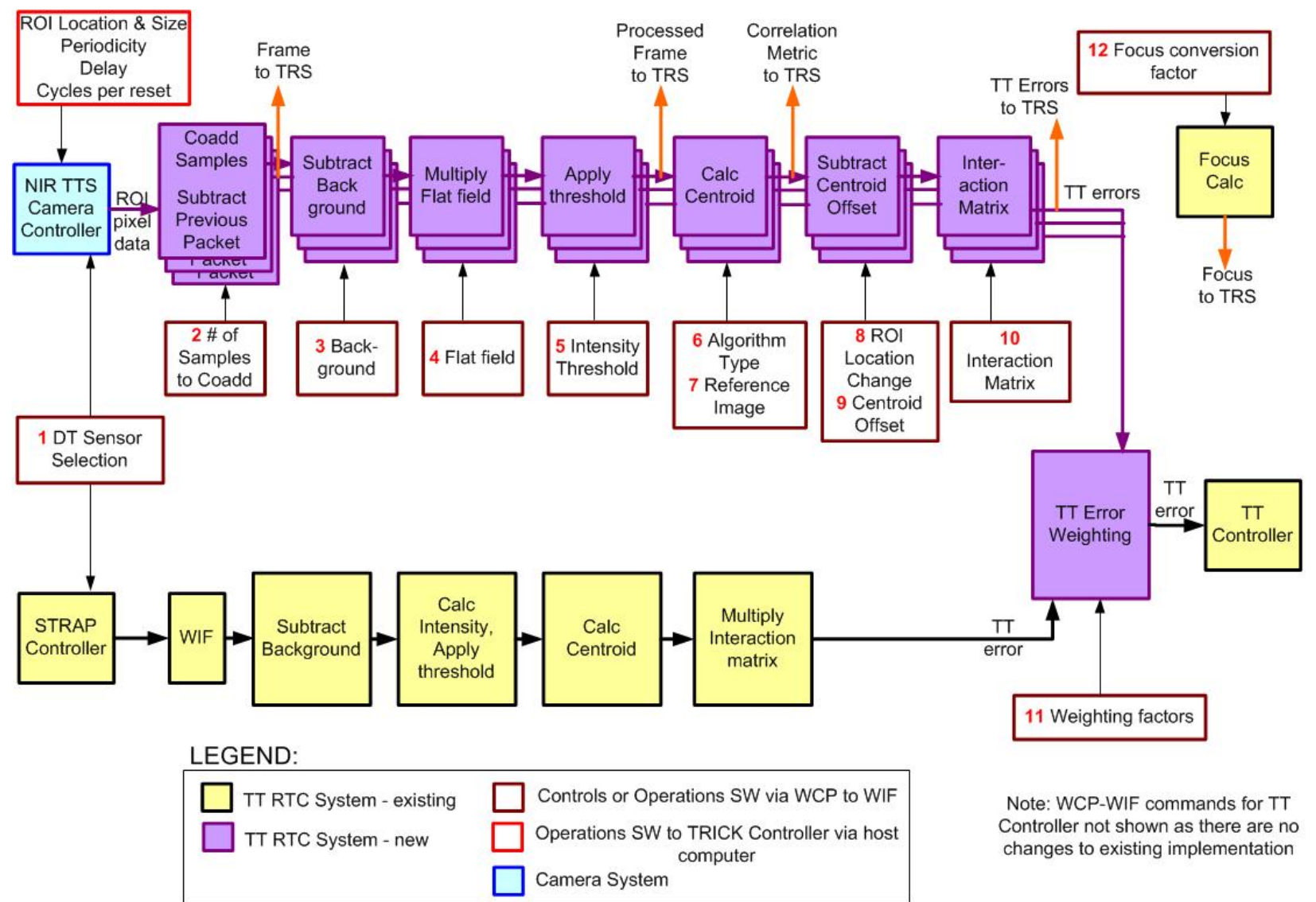

Note: WCP-WIF commands for TT Controller not shown as there are no changes to existing implementation

Figure 8: Real-time control processing steps

\subsection{Opto-mechanical System}

The opto-mechanical system consists of (1) a vertical stage holding up to three pickoff optics, (2) a focus stage for the camera, and (3) the modifications to the AO bench covers to support the new tip-tilt sensor system. Two choices of pickoff optic, both manufactured by Custom Scientific, are currently available: (1) a K-band reflective dichroic that transmits the shorter wavelengths and (2) an annular gold-coated optic with an H-band reflective/K-band transmissive 
dichroic at its center. The purpose of the latter optic is to optimally support K-band integral field spectroscopy by allowing $\geq 35$ " off-axis tip-tilt guide stars to be used in Ks-band. The measured transmission loss to OSIRIS with either dichroic in the science path is $7 \%$.

\subsection{Controls System}

The control loops associated with tip-tilt (i.e. image motion) are shown in Figure 9. The responsibilities of the real-time control (RTC), camera, controls and operations software are indicated by different color boxes (purple, blue, green and orange, respectively). The light path through the existing active optics (telescope, rotator, tip-tilt mirror [TTM], deformable mirror [DM]) to the OSIRIS science instrument is shown in red. The control loop signal paths are shown in green. The real-time loop is from the readout of a ROI by the camera controller to the RTC where the tip-tilt is computed, and hence to the TTM. The controls system is responsible for four of the boxes shown in Figure 9: (1) the existing offloading of the TTM to the telescope pointing; (2) setting the focus position of the NIR TTS to be conjugate to the science instrument focal plane and setting the pickoff stage and NIR TTS camera filter to be compatible with the OSIRIS setup; (3) computing the differential atmospheric refraction between the TTS and OSIRIS wavelengths; and (4) coordinating the ROI position to be used by the camera and the centroid offset to be used by the RTC.

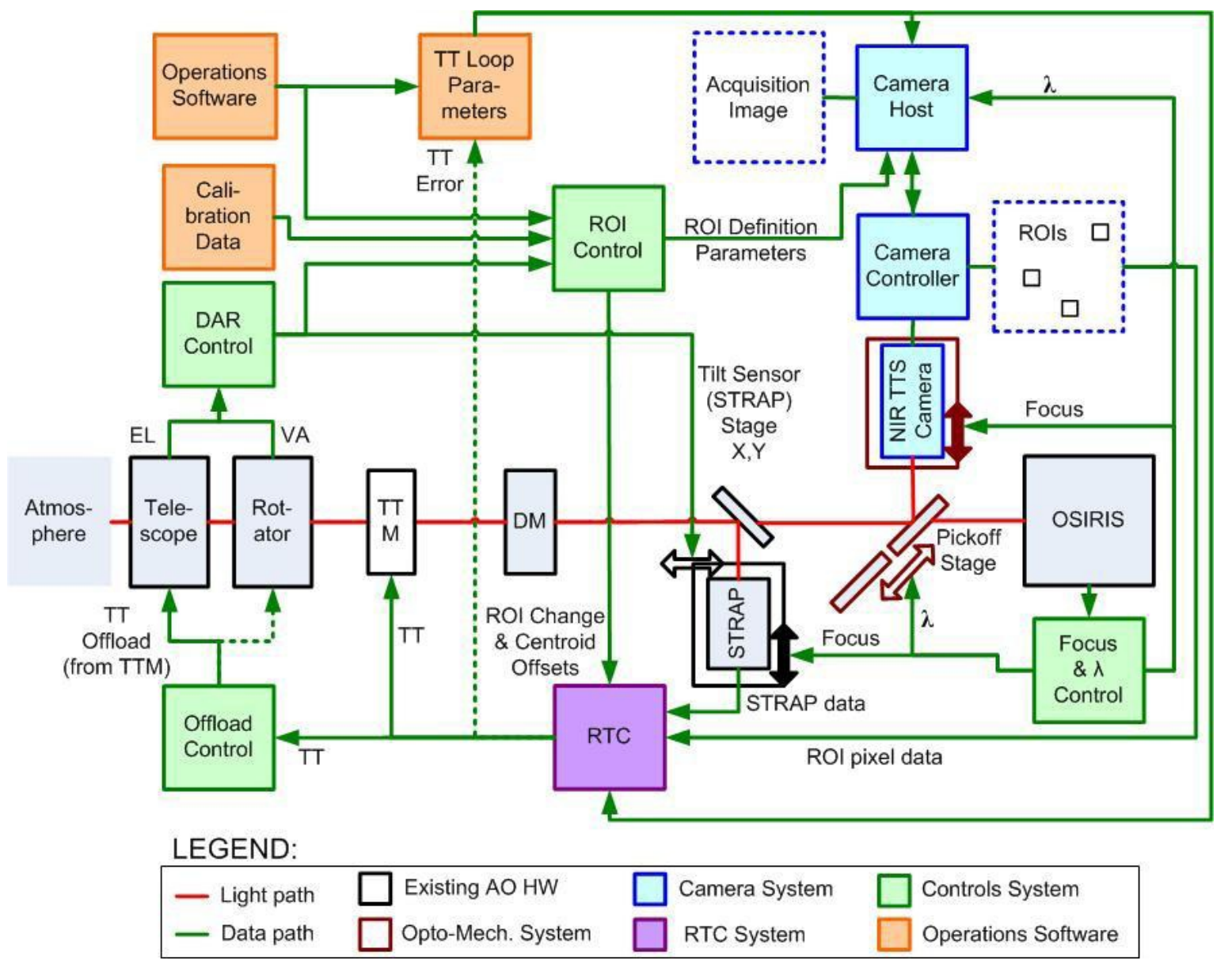

Figure 9: Tip-tilt (TT) control loops

\subsection{Operations Software System}

The Operations software is responsible for setting the NIR TTS camera and RTC parameters as shown in Figure 8 and Figure 9. The one exception is changing the ROI location and the centroid offsets (steps 8 and 9 in Figure 8 ) which are instead provided by the Controls system.

The key camera parameters to be set by the Operations software are the ROI size and position, the number of nondestructive reads to be coadded and the number of readout cycles per reset. When more than one ROI is to be used the 
number of ROIs will also need to be set. The ROI location for the NGS is chosen to position the science object at the appropriate location on OSIRIS.

The higher order (i.e. deformable mirror) loop is closed on the LGS first. The tip-tilt loop can then be closed on the resultant near-diffraction limited image core of the NGS. The ROI size can start out as 16x16 pixels during acquisition and is shrunk to $4 \times 4$ pixels or $2 \times 2$ pixels as the tip-tilt residual is reduced.

The number of readouts to coadd (see Figure 6) is selected to optimize the tip-tilt performance. More reads generally reduce the measurement error at the expense of bandwidth error. The effective frame rate associated with the predicted optimal number of coadds is shown in Figure 10. The optimal number of coadds for the $4 x 4$ pixel ROI case in Figure 10 ranges from 7 for $\mathrm{K}=10$, to 64 for $\mathrm{K}=16$.

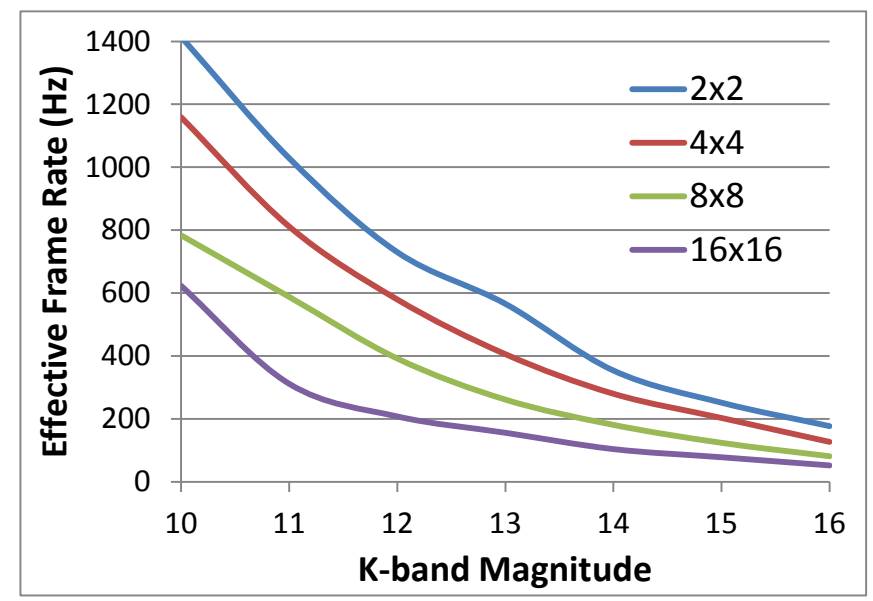

Figure 10: Effective frame rate versus K-band magnitude at zenith for an on-axis guide star and for ROI sizes ranging from $2 \times 2$ to $16 x 16$ pixels.

At least one correction cycle is lost after each reset since two sets of coadds are needed to do a subtraction. The number of resets is minimized by ensuring that the integrated flux per pixel is just under saturation before resetting the detector. The impact of a reset is minimized by requiring the number of cycles per reset to be an integer number of coadds. The cycles per reset corresponding to the $4 \times 4$ pixel ROI case in Figure 10 ranges from 511 at $10^{\text {th }}$ magnitude to 16211 at $14^{\text {th }}$ magnitude after which it is not increased (in order for the time between resets to be at most $2 \mathrm{~s}$ ).

The user must decide between using the existing visible and new near-infrared tip-tilt sensor. Table 1 provides a comparison between these two sensors.

Table 1: Tip-tilt sensor comparison.

\begin{tabular}{|c|c|c|}
\hline Comparison Criteria & STRAP & NIR TTS \\
\hline OSIRIS modes supported & All & All but K-band imaging \\
\hline Throughput loss & None & $7 \%$ \\
\hline Image quality degradation & None & Negligible \\
\hline Field of Regard diameter & 90 to $120^{\prime \prime}$ & $0.05 "$ \\
\hline Pixel size & $1.4^{\prime \prime}$ & H=16 or K=16 \\
\hline Limiting magnitude & R=19 & K-reflective dichroic \\
\hline \multicolumn{2}{|c|}{ For Science short of K-band } \\
\hline Additional optics in OSIRIS path & None & Ks-band \\
\hline Tilt Sensor Wavelength & R-band & Forband IFS Science \\
\hline Additional optics in OSIRIS path & None & $\begin{array}{c}\text { Gold annular mirror with H- } \\
\text { reflective dichroic at center }\end{array}$ \\
\hline Tilt Sensor Wavelength & R-band & $\begin{array}{l}\text { Ks-band for NGS > 35" } \\
\text { H for < 35" off-IFS-axis }\end{array}$ \\
\hline
\end{tabular}




\section{PERFORMANCE PREDICTIONS}

The predicted residual one-dimensional rms tip-tilt error for $\mathrm{H}$ and Ks-band tip-tilt sensing, and for the existing STRAP sensor are shown in Figure 11. The advantage of Ks-band sensing for large sky fraction is very clear. H-band sensing has an advantage over STRAP for the low sky coverage case but the H-band Strehl ratio is simply too low when moving far off-axis as required for high sky coverage.

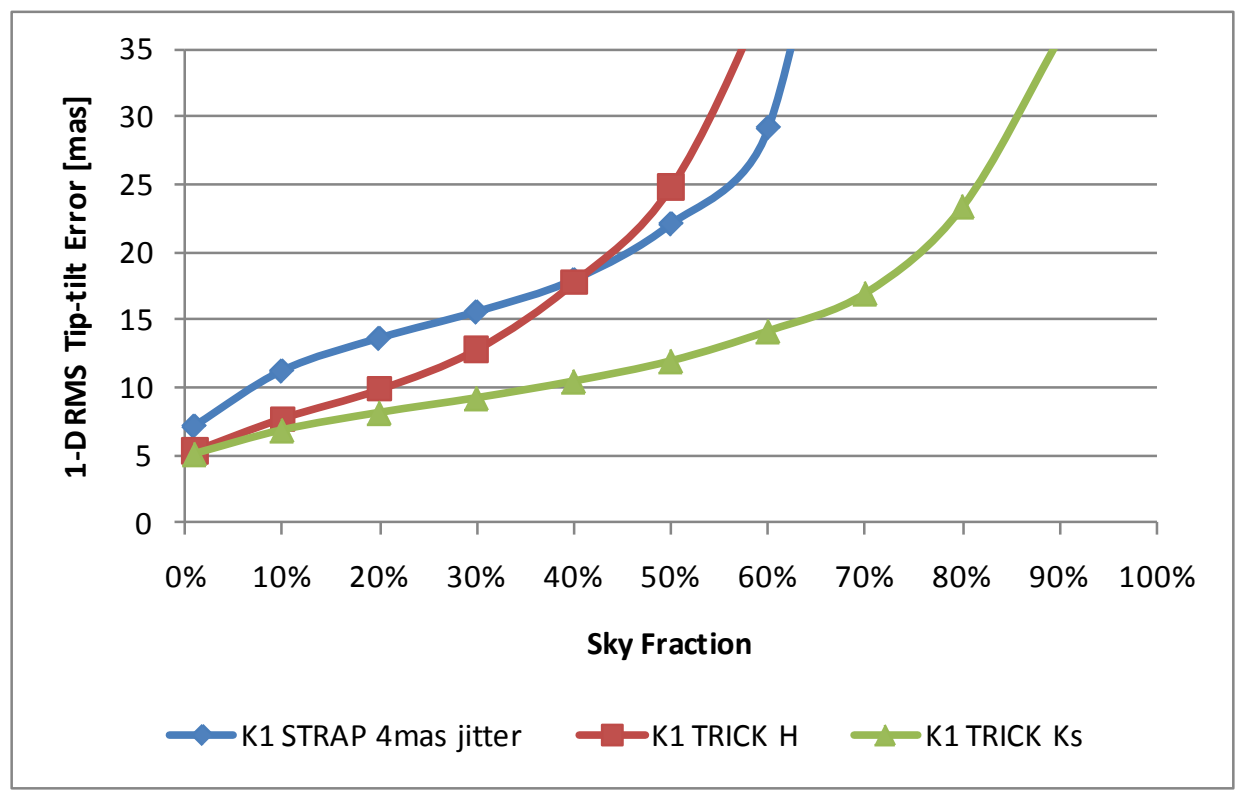

Figure 11: Residual 1-dimensional rms tip-tilt error for the NIR tip-tilt sensor (TRICK) and STRAP.

Tip-tilt performance depends on tip-tilt star color as shown in Figure 12. STRAP outperforms NIR tip-tilt sensing for stars of $\mathrm{G}$ spectral type or earlier. The vast majority of main sequence stars are however $\mathrm{M}$ spectral class for which the NIR sensor performance, in Ks and H, significantly outperforms STRAP. As can be seen from this plot the performance predictions are very dependent on star color. The $\mathrm{M}$ stars are $\sim 0.25$ magnitudes brighter in $\mathrm{K}$ than $\mathrm{H}$.

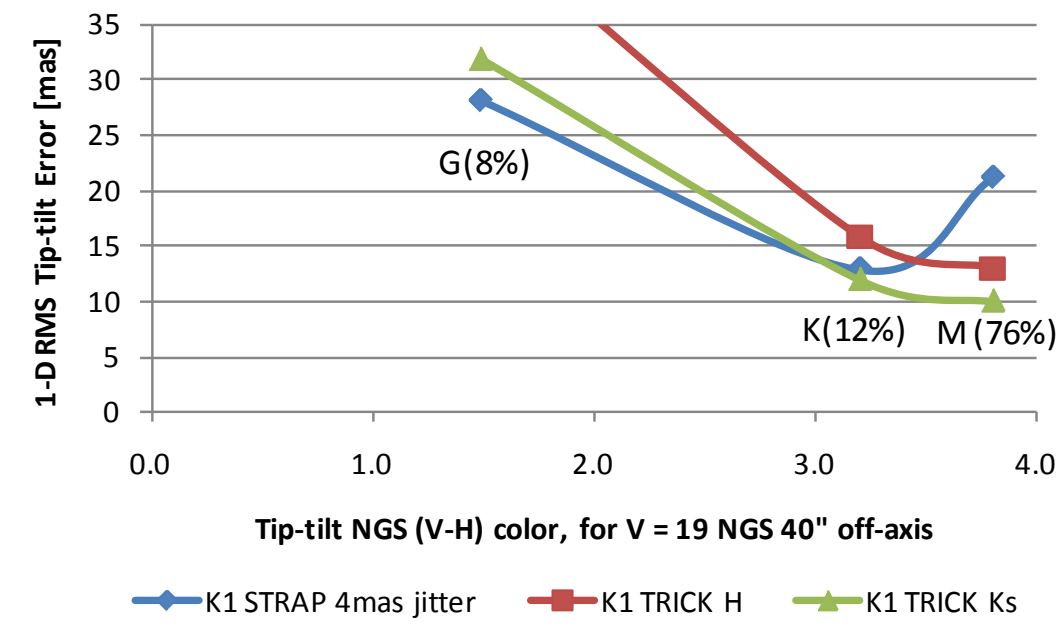

Figure 12: Tip-tilt performance for a V = 19 tip-tilt star located 40" off-axis, assuming different spectral type stars. The number in parentheses is the fraction of all main sequence stars of that type [9]. 
There are two impacts of bad pixels. The first is making some areas of the detector unavailable for centroiding. The second is to impact the computed centroid. This impact is mitigated by excluding bad pixels from the centroid computation by setting their gain to zero in the flat field map. A bad pixel in an ROI will introduce a centroid shift for that ROI, and hence an offset on OSIRIS. For example, for a Strehl ratio of 0.25 the centroid shift is predicted to be between 4 mas and 8.5 mas if the bad pixel is in the central 2x2 pixels of a $4 \times 4$ pixel ROI or by 1.3 mas to 1.7 mas if the bad pixel is in the outer $4 \times 4$ pixels. The shift grows as the Strehl ratio is reduced.

\section{INITIAL PERFORMANCE RESULTS}

\subsection{NIR Camera Image Quality}

A multi-hole mask and sub-pixel dithering were used at Caltech to measure the image quality of the camera. Over the central 49"x46"the Ks-band Strehl ratio was found to range between $91.6 \%$ and $95.5 \%$ with an average of $94.1 \%$; this performance matched the expected design performance within the measured sub-field. Five different mask locations were used to measure the H-band performance which was found to be $2.6 \%$ poorer than the nominal camera design. The measured average $\mathrm{H}$-band Strehl was $88 \%$ with a $1 \sigma$ variance of $4 \%$.

The NIR camera Ks-band image size measured with a single mode fiber at the input of the AO system and a flat deformable mirror was measured to be $\sim 56$ mas FWHM; this can be reduced to $\sim 52$ mas by removing astigmatism with the deformable mirror. Using phase diversity, again with the AO input fiber as a source, $\sim 180 \mathrm{~nm}$ of rms wavefront error was measured for the AO system plus NIR camera (primarily $\sim 164 \mathrm{~nm}$ of $0^{\circ}$-astigmatism and $\sim 45 \mathrm{~nm}$ of $\mathrm{x}$-coma).

A pair of on-sky images at different field positions is shown in Figure 13.

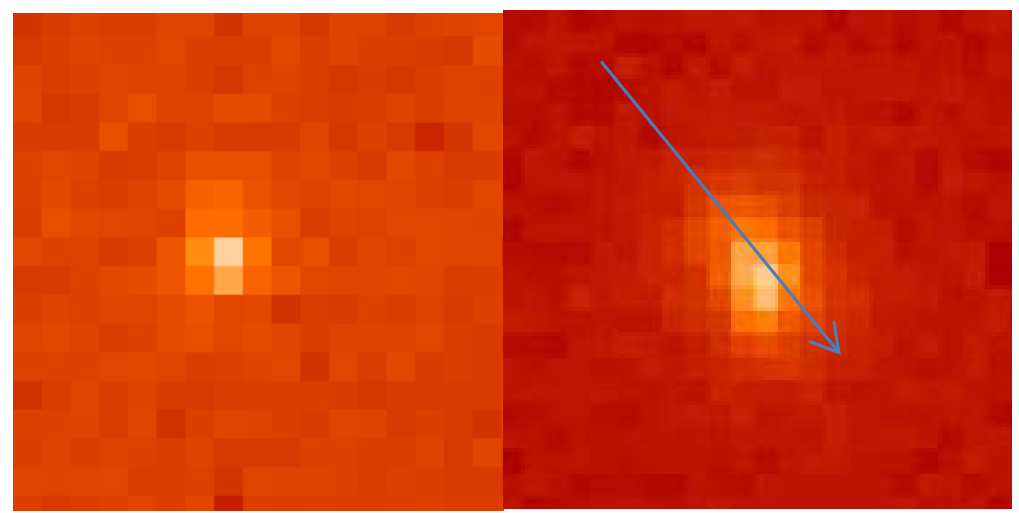

Figure 13: On-sky Ks-band images on the NIR camera with the NGS AO loops closed on the wavefront sensor. The left and right images are 9.3" and 52" off-axis with respect to the wavefront sensor, respectively. The FWHM of the two images are 83 mas and 130 mas, respectively. The right image provides an example of the spot elongation due to tilt anisoplanatism (the arrow points to the NGS used by the wavefront sensor).

Field distortion and curvature have not yet been measured. The optical design predicts image displacements of up to 0.9 " or 18 pixels at the edge of the field while the field curvature effects are predicted to be negligible.

\subsection{NIR Camera Ks-band Zero Point Magnitude}

The stellar magnitude can be determined by $m=-2.5 \log F+Z P$ where the stellar flux, $F$, is in ADU/s and ZP is the zero point magnitude. Six sources in the M35 cluster were used to obtain an initial Ks-band zero point for the NIR camera. The preliminary result is $\mathrm{ZP}_{\mathrm{Ks}}=26.18 \pm 0.02$. For comparison the $\mathrm{ZP}_{\mathrm{K}}$ for the $\mathrm{AO}$ science instrument NIRC2 is 24.63.

\subsection{Offsetting}

Offsetting of the image on OSIRIS can be performed in two ways with the NIR tip-tilt sensor: by moving the ROI to a new location or by introducing a centroid offset. The former moves the image in units of ROI pixels while the latter moves the image in fractions of a pixel. Daytime tests have been performed for both of these offsets. The measured image shift is (1) $2.40 \pm 0.08$ OSIRIS pixels per NIR tip-tilt sensor pixel, and (2) $2.10 \mathrm{X} \pm 0.25$ OSIRIS pixels for centroid offsets of $\mathrm{X} \leq 0.75$ pixels when using a $4 \times 4$ pixel ROI. The results of the latter test are shown in Figure 14 . There is no apparent correlation between this $2.10 \mathrm{X}$ ratio and the magnitude of the requested centroid offset. 


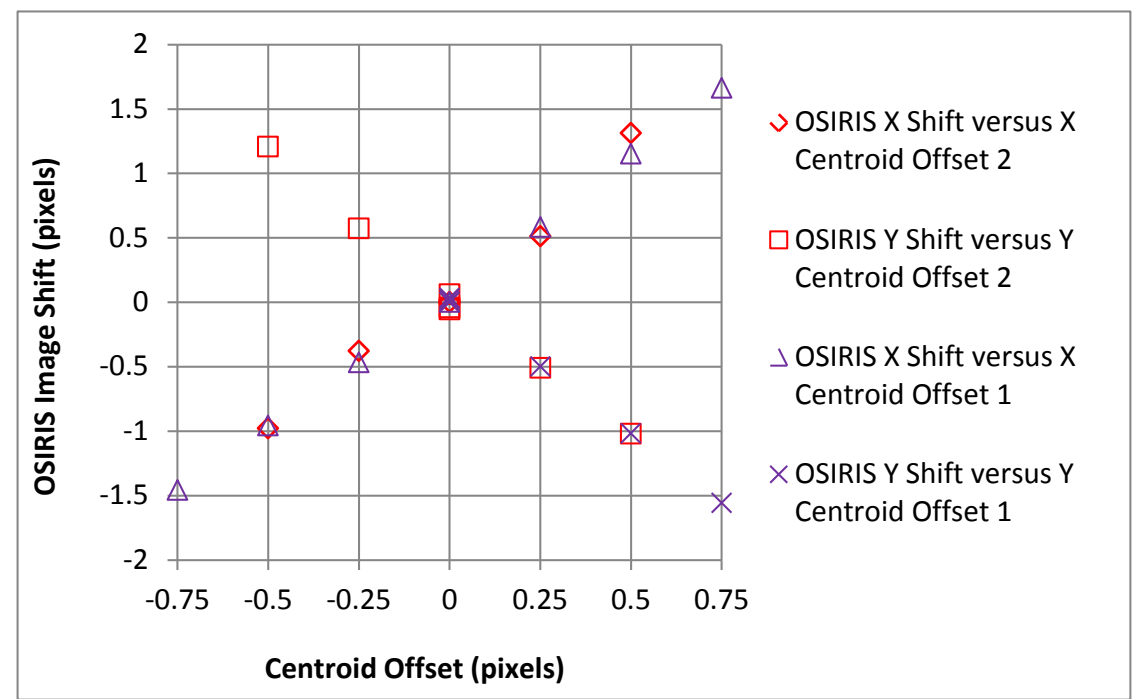

Figure 14: The measured image shift on OSIRIS versus the requested centroid offset for a sample $4 \times 4$ pixel ROI.

\subsection{Tip-Tilt Performance}

One night of on-sky performance data has been acquired to date. Binary star pairs were used where the faint star was positioned near the center of the OSIRIS imager. The K-band reflective dichroic was placed in the science path and OSIRIS was configured with an Hn3 filter $(1.64 \mu \mathrm{m})$ and the NIR sensor used its Ks-band filter $(2.15 \mu \mathrm{m})$. Initially both the tip-tilt and deformable mirror loops were closed on the off-axis bright star. The tip-tilt loop was then closed, using the NIR tip-tilt sensor, on either the bright off-axis star or the fainter on-axis star. The observation parameters are tabulated in the first ten columns of Table 2. For example, for the first target (TYC 2534-735-1) the deformable mirror loop was always closed on the $\mathrm{R}=9.9$ star located 15.1" off-axis. The tip-tilt loop was alternately closed on the wavefront sensor (WFS) using the $\mathrm{R}=9.9$ star or on the NIR sensor using either the same off-axis star $(\mathrm{K}=8.5)$ or the on-axis star $(\mathrm{K}=12.0)$. Note that faint TYC 2539-1069-1 star is very red with $\mathrm{R}=19.7$ and $\mathrm{K}=15.25$, too faint for STRAP but doable for the NIR sensor even at an elevation of $38^{\circ}$.

For reference the K-band Strehl ratio and FWHM after calibration on the reference single mode fiber at the input to the AO system were 0.78 and 44.6 mas, respectively (image sharpening was done in the K-band through the K-band dichroic in order not to saturate OSIRIS).

The measured Strehl ratio and FWHM on OSIRIS are listed in the last two columns of Table 2. These are also plotted in Figure 15 versus R-magnitude for the WFS and K-magnitude for the NIR sensor. If the same NGS is used for tip-tilt, the OSIRIS image quality is as good or better with the NIR sensor as with the WFS (see the bright off-axis star data points in the Figure 15 ovals). The degradation in performance when switching from using the bright off-axis NGS to using the faint on-axis NGS for NIR tip-tilt sensing is due to two factors: the tip-tilt NGS has a lower Ks-band Strehl ratio due to isoplanatism ( $\sim 1 / 2$ at a separation of $\sim 15$ " from the NGS used for high order correction) and it is considerably fainter.

Table 2: H-band FWHM versus test set up parameters.

\begin{tabular}{|c|c|c|c|c|c|c|c|c|c|c|c|}
\hline \multirow{2}{*}{$\begin{array}{l}\text { Target } \\
\text { TYC \# }\end{array}$} & \multicolumn{2}{|c|}{ DM Star } & \multicolumn{3}{|c|}{ Tip-Tilt Star } & \multirow{2}{*}{$\begin{array}{l}\text { ro } \\
\text { (m) }\end{array}$} & \multirow{2}{*}{$\begin{array}{c}\text { Elevation } \\
\left({ }^{\circ}\right)\end{array}$} & \multirow[b]{2}{*}{ Sensor } & \multirow{2}{*}{\begin{tabular}{|c|} 
OSIRIS \\
Exposure \\
(s)
\end{tabular}} & \multicolumn{2}{|c|}{ Hn3 } \\
\hline & R-mag & $\begin{array}{c}\text { Off-axis } \\
\text { (") }\end{array}$ & R-mag & K-mag & $\begin{array}{c}\text { Off-axis } \\
\text { (") }\end{array}$ & & & & & $\begin{array}{c}\text { Strehl } \\
\text { ratio }\end{array}$ & $\begin{array}{l}\text { FWHM } \\
\text { (mas) }\end{array}$ \\
\hline \multirow{3}{*}{$\begin{array}{c}2534-735- \\
1\end{array}$} & \multirow{3}{*}{9.9} & \multirow{3}{*}{15.1} & \multirow{2}{*}{9.9} & \multirow{2}{*}{8.5} & \multirow{2}{*}{15.1} & \multirow{3}{*}{0.22} & \multirow{2}{*}{74 to 62} & WFS & 2 & 0.15 & 44.7 \\
\hline & & & & & & & & NIR & 2 & 0.17 & 45.9 \\
\hline & & & 13.7 & 12.0 & 0 & & 62 to 58 & NIR & 2 & 0.11 & 60.9 \\
\hline \multirow{2}{*}{$\begin{array}{c}2539- \\
1069-1\end{array}$} & \multirow{2}{*}{10.1} & \multirow{2}{*}{11.3} & 10.1 & 8.8 & 11.3 & \multirow{2}{*}{0.16} & 56 to 45 & WFS & 10 & 0.05 & 55.9 \\
\hline & & & 19.7 & 15.3 & 0 & & 44 to 38 & NIR & 10 & 0.03 & 82.4 \\
\hline \multirow{2}{*}{$\begin{array}{c}2650-535- \\
1\end{array}$} & \multirow{2}{*}{10.0} & \multirow{2}{*}{17.4} & 10.0 & 8.8 & 17.4 & \multirow{2}{*}{0.20} & \multirow{2}{*}{73 to 74} & WFS & 10 & 0.10 & 56.1 \\
\hline & & & 15.7 & 15.1 & 0 & & & NIR & 10 & 0.07 & 74.9 \\
\hline
\end{tabular}



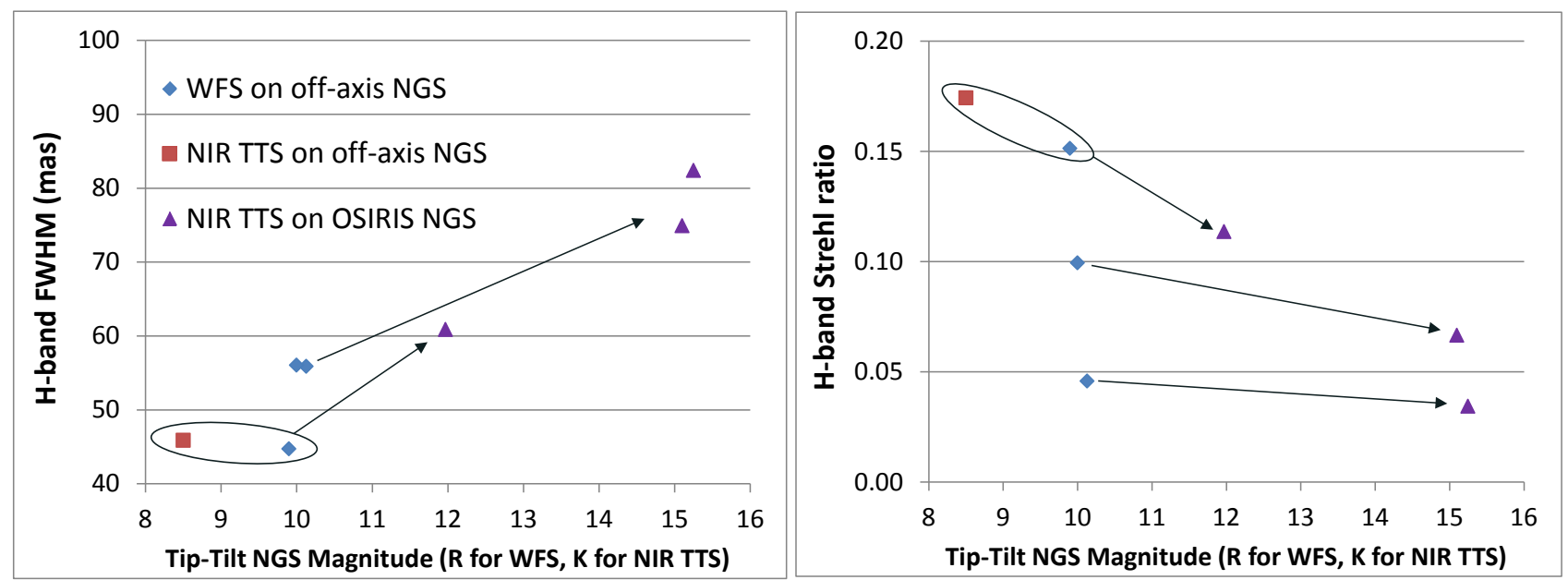

Figure 15: H-band FWHM (left) and Strehl ratio (right) at the center of OSIRIS versus magnitude of the faint onaxis or bright off-axis NGS used for tip-tilt (the deformable mirror loop was closed on the bright off-axis NGS). The points within the oval are for the same off-axis NGS. The arrows point from the off-axis NGS data points to the corresponding on-axis NGS data points.

Each Strehl ratio and FWHM data point in Table 2 and Figure 15 is the average of multiple exposures. For example, the FWHM for each 10 s OSIRIS image of TYC 2650-535-1 is shown in Figure 16. In this sequence the tip-tilt loop was alternately closed between the WFS with a tip-tilt loop gain of 0.3 and the NIR sensor with the tip-tilt loop gains and number of coadds listed in the figure legend. The performance was not very sensitive to these choices of NIR tip-tilt correction parameters. The 0.001 gain case is essentially the no tip-tilt correction case, with a FWHM of 132 mas.

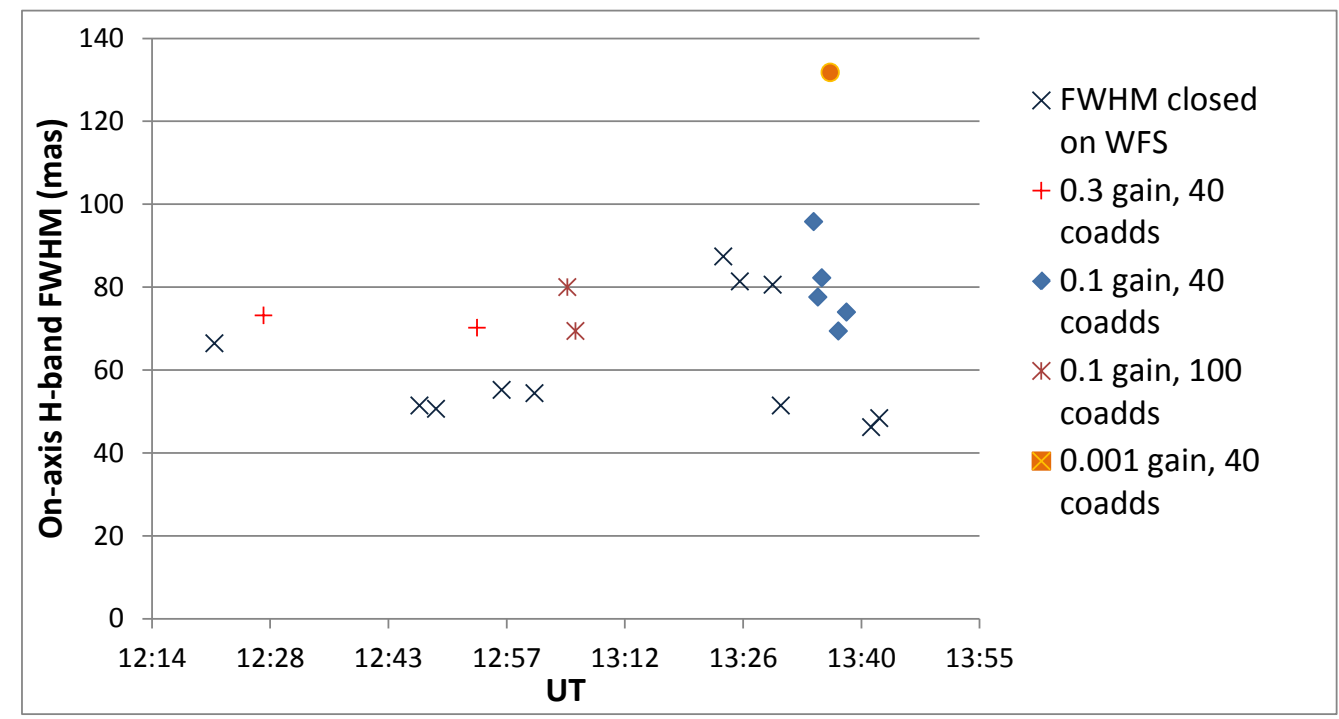

Figure 16: H-band FWHM on OSIRIS versus time for the target TYC 2650-535-1 as either the WFS or NIR sensor were used for tip-tilt sensing. The legend includes the tip-tilt loop gains and number of coadds used for NIR tip-tilt correction.

\section{CONCLUSION AND NEAR-TERM PLANS}

A NIR tip-tilt sensor has been implemented with the Keck I LGS AO system. The system is based on the low noise demonstrated to be achievable with multiple reads of regions of interest on a H2RG detector. The performance predictions and initial on-sky tests indicate that the sky coverage of the Keck I system will be significantly increased. 
Additional on-sky testing and commissioning time is scheduled for August to October 2014. Pending successful completion of the commissioning and handover to operations, the NIR tip-tilt sensor will be made available for sharedrisk science with the Keck I LGS AO system.

The initial offered mode of the NIR sensor uses a single ROI for tip-tilt sensing. We plan to test and commission the following additional modes within the following year if they prove to be scientifically useful:

- The use of multiple ROIs to reduce tilt anisoplanatism.

- The use of a correlation algorithm to measure the image position (versus the current centroid algorithm).

- The use of a single ROI to also provide focus information (at higher bandwidth than the existing low bandwidth wavefront sensor that reads out every $10 \mathrm{~s}$ to $180 \mathrm{~s}$ depending on the NGS magnitude).

- $\quad$ Parallel operation of STRAP with the NIR tip-tilt sensor.

- Improved planning and optimization tools.

\section{ACKNOWLEDGEMENTS}

This material is based in part upon work supported by the National Science Foundation under Grant No. AST-1007058. We would like to acknowledge the technical contributions of Jon Chock, Liz Chock, Shui Kwok, Sudha Laven, Scott Lilley, Jeff Mader, Olivier Martin, Bill Randolph, Kevin Tsubota and Ed Wetherell at WMKO, Ernest Croner at Caltech, and Antonin Bouchez at GMT. We would also like to thank our science team of Tommaso Treu at University of California (UC) Santa Barbara and Mark Morris at UC Los Angeles.

The W. M. Keck Observatory is operated as a scientific partnership among the California Institute of Technology, the University of California, and the National Aeronautics and Space Administration. The Observatory was made possible by the generous financial support of the W. M. Keck Foundation.

The authors wish to recognize and acknowledge the very significant cultural role and reverence that the summit of Mauna Kea has always had within the indigenous Hawaiian community. We are most fortunate to have the opportunity to conduct observations from this mountain.

\section{REFERENCES}

[1] Wizinowich, P. et al., "First Light Adaptive Optics Images from the Keck II Telescope: A New Era of High Angular Resolution Imagery,” PASP 112, 315-319 (2000).

[2] Wizinowich, P. et al., “The W. M. Keck LGS AO System: Overview,” PASP 118, 297-309 (2006).

[3] van Dam, M. et al., "The W. M. Keck LGS AO System: Performance Characterization," PASP 118, 310-318 (2006).

[4] Chin, J. et al., "Keck I Laser Guide Star Adaptive Optics System,” SPIE Proc. 8447, 8 (2012).

[5] Wizinowich, P., “Astronomical Science with Adaptive Optics at W. M. Keck Observatory,” PASP 125, 798-808 (2013).

[6] Larkin, J. et al., “OSIRIS: A diffraction limited integral field spectrograph for Keck,” New Astronomy Reviews 50, 362-364 (2006).

[7] Smith, R. and Hale, D., "Bad pixel mapping,” SPIE Proc. 9154-16 (2014).

[8] Smith, R. and Hale, D., "Read noise for a $2.5 \mu \mathrm{m}$ cutoff Teledyne H2RG at 1-1000Hz frame rates," SPIE Proc. 8453, $84530 Y(2012)$.

[9] LeDrew, G., “The Real Starry Sky,” JRASC 95, 32 (2001). 